D. Fehrle, C. Grabianowski and G. Jones (Coordinators) (grant HL074408). University of Alabama at Birmingham, Birmingham, AL, USA: W.C. Bailey and J.A.D. Cooper (Co-Principal Investigators), M.T. Dransfield, L.B. Gerald and P. O’Reilly (Investigators), S. Tidwell (Coordinator) (grant HL074418). University of California, San Francisco, CA, USA: S.C. Lazarus (Principal Investigator), H.A. Boushey and P.G. Woodruff (Investigators), M. Birch, R. Sakurai, K. Schardein, M. Dyjak and C. Nguyen (Coordinators) (1U10-HL074431). University of Maryland, Baltimore, MD, USA: S.M. Scharf (Principal Investigator), M. Alattar, P. Amelung, M. Cowan, J. Hanson, J. Hasday, A. Iacono, C. Shanholtz, N. Todd and A. Verceles (Investigators), T. Fitzgerald, W. Bell-Farrell and P. Wood (Coordinators) (grant HL074441 and GCRC grant RR16500). University of Michigan, Ann Arbor, MI, USA: F.J. Martinez (Principal Investigator), J.L. Curtis, M.K. Han, K.R. Flaherty, T. Standiford, S.E. Gay and T.E. Sisson (Investigators), D. Thompson, L. McCloskey, M. Christensen and D. White (Coordinators) (grant HL074422). University of Pittsburgh, Pittsburgh, PA, USA: F. Sciurba (Principal Investigator), J. Bon (Investigator), L. Kniolek, M. Pitaro and R. Folger (Coordinators) (grant HL074439 and GCRC grant RR00056). University of Minnesota (Data Coordinating Center), Minneapolis, MN, USA: J.E. Connett (Principal Investigator), N.R. Anthonisen (Steering Committee Chair), C. Wendt (Co-Principal Investigator), M. Skeans, W. Patrek, H. Voelker and S. Harnden (Coordinators) (grant 1U10- HL074424). Data and Safety Monitoring Board: B.B. Bender, S.F. Kelsey, J.R. Landis, B. Phillips, G.M. Turino, R. Veatch, A. Waldo, C. Champlin and A. Wanner (all National Heart, Lung, and Blood Institute, Bethesda, MD, USA). Protocol Review Committee: H.W. Kelly (University of New Mexico-Health Sciences Center, Albuquerque, NM, USA), J. Maurer (The Cleveland Clinic Foundation, Cleveland, OH, USA), A.J. McSweeny (Northwestern University Medical School, Chicago, IL, USA), R.M. Senior (Washington University School of Medicine, St Louis, MO, USA), E.A. Thom (George Washington University, Rockville, MD, USA), P.D. Wagner (University of California, San Diego. San Diego, CA, USA), R.L. ZuWallack (Saint Francis Hospital and Medical Center, Hartford, CT, USA). National Heart, Lung, and Blood Institute, Bethesda, MD, USA: G. Weinmann (Deputy Director, Division of Lung Diseases), T. Croxton (Director, Airway Biology and Disease Program), A. Punturieri (Program Officer) and M.P. Stylianou (Biostatistician).

\title{
References
}

1 Albert RK, Connett J, Bailey WC, et al. Azithromycin for prevention of exacerbations of COPD. N Engl J Med. 2011; 365: 689-698.

2 Niewoehner DE, Rice K, Cote C, et al. Prevention of exacerbations of chronic obstructive pulmonary disease with tiotropium, a once-daily inhaled anticholinergic bronchodilator: a randomized trial. Ann Intern Med 2005; 143: 317-326.

3 Aggarwal BB. Tumour necrosis factors receptor associated signalling molecules and their role in activation of apoptosis, JNK and NF-кB. Ann Rheum Dis 2000; 59: Suppl. 1, i6-i16.

4 Hijdra D, Vorselaars AD, Grutters JC, et al. Differential expression of TNFR1 (CD120a) and TNFR2 (CD120b) on subpopulations of human monocytes. J Inflamm (Lond) 2012; 9: 38.

5 Aderka D, Engelmann H, Wysenbeek AJ, et al. The possible role of tumor necrosis factor (TNF) and its natural inhibitors, the soluble-TNF receptors, in autoimmune diseases. Isr J Med Sci 1992; 28: 126-130.

6 Aderka D, Engelmann H, Maor Y, et al. Stabilization of the bioactivity of tumor necrosis factor by its soluble receptors. J Exp Med 1992; 175: 323-329.

7 Takabatake $\mathrm{N}$, Nakamura $\mathrm{H}$, Abe S, et al. The relationship between chronic hypoxemia and activation of the tumor necrosis factor-alpha system in patients with chronic obstructive pulmonary disease. Am J Respir Crit Care Med 2000; 161: 1179-1184.

8 Groenewegen $\mathrm{KH}$, Dentener MA, Wouters EF. Longitudinal follow-up of systemic inflammation after acute exacerbations of COPD. Respir Med 2007; 101: 2409-2415.

9 Nozaki N, Yamaguchi S, Shirakabe M, et al. Soluble tumor necrosis factor receptors are elevated in relation to severity of congestive heart failure. Jpn Circ J 1997; 61: 657-664.

10 Osika E, Cavaillon JM, Chadelat K, et al. Distinct sputum cytokine profiles in cystic fibrosis and other chronic inflammatory airway disease. Eur Respir J 1999; 14: 339-346.

\section{Pulmonary embolism risk stratification: where are we heading?}

\author{
To the Editor:
}

We read with great interest the paper by SANCHEZ et al. [1], in which the authors suggested that biomarkers and echocardiography findings would provide additional prognostic information to traditional risk models, such as the pulmonary embolism severity index (PESI), in normotensive acute pulmonary embolism (PE) patients. Patients were assigned to low- (PESI I-II), intermediate- (PESI III-IV) and high-risk classes (PESI $\mathrm{V}$ ) and further stratified according to right ventricle dysfunction markers (right ventricle/left ventricle ratio, and troponin and brain natriuretic peptide (BNP) levels). The authors reported that low-risk PESI patients without any right ventricle dysfunction markers had a significantly lower risk for adverse events than low-risk patients with abnormal right ventricle/left ventricle ratio, troponin and/or BNP levels. However, the same results were not found for the remaining PESI classes (intermediate and high risk). 
Reliable risk stratification represents the cornerstone of PE management, which should be capable of recognising patients at high risk of death, who could receive specific therapeutic interventions, and those suitable for outpatient management, with important savings in healthcare costs. Although the PESI score has a proven high discriminative performance in the identification of low-risk patients, outperforming other validated PE risk models [2,3], some authors have recently demonstrated that the 48-h recalculation of PESI can more accurately identify those eligible for outpatient management [4]. Serial calculation of PESI and a decision on potential discharge at the 24-48-h mark, as an early evaluation of treatment response, might be a comprehensive risk stratification strategy with high certainty of safety.

Although the combination of validated PE risk scores with other easily available illness severity markers may be a more suitable approach to evaluate prognosis of this complex condition, early adverse events were still reported by SANCHEZ et al. [1] in the very low risk class (PESI I-II and normal right ventricle dysfunction markers).

One of the markers of right ventricle dysfunction used was BNP, which was considered abnormal when $>78.5 \mathrm{ng} \cdot \mathrm{L}^{-1}$. This is an appropriate cut-off point to rule-out right heart failure [5]; however, it lacks adequate specificity to be used as an isolated heart dysfunction marker since BNP varies with other conditions, such as renal failure or chronic obstructive pulmonary disease. BNP can also be elevated in previously known left heart failure, a variable already considered within PESI, in which case it would not reflect PE severity. Moreover, BNP predictive value may not be independent from echocardiographic findings of right ventricle dysfunction. Accordingly, the higher concentrations of BNP reported in the highrisk group (PESI V) did not correlate with higher degrees of right ventricle dilatation on echocardiogram and was not an independent predictor of adverse outcomes.

In the Low Risk Pulmonary Embolism Decision (LR-PED) rule derivation sample [6], comprising haemodynamically stable patients without right ventricle dysfunction (based on N-terminal pro-BNP and echocardiogram), the presence of a troponin I level $\geqslant 0.1 \mathrm{ng} \cdot \mathrm{mL}^{-1}$ was associated with an increased 1 -month mortality ( $14.3 \%$ versus $26.2 \%$ ), although the difference did not reach statistical significance due to the low number of events. Furthermore, $59.4 \%$ of patients with troponin $\mathrm{I}<0.1 \mathrm{ng} \cdot \mathrm{mL}^{-1}$, with a simplified PESI $\geqslant 1$ (high-risk class according to this risk model) had a $12.5 \% 1$-month mortality. These findings suggest that, although troponin I and other right ventricle dysfunction markers may add prognostic value, PESI or simplified PESI still prevail over the former parameters.

Other biomarkers with independent prognostic value may merit inclusion in future refined risk analysis. The LR-PED rule included several clinical variables and acute phase markers (glycaemia, C-reactive protein, troponin and creatinine at admission) and apparently presented higher sensitivity and negative predictive value than the GENEVA score and simplified PESI risk schemes, allowing improved risk reclassification of truly low-risk PE patients. Furthermore, the same working group proposed that the presence of atrial fibrillation has independent predictive power and should also be considered in PE prognosis, suggesting that heart rhythm might be as important as heart rate in this complex condition [7].

Regarding echocardiographic evaluation of acute right ventricle pressure overload, right ventricle/left ventricle ratio may not have high sensitivity for the detection of subclinical right ventricle systolic dysfunction. However, speckle tracking imaging is an angle-independent approach for assessment of both global and regional right ventricle function, potentially outperforming the classic parameters of right ventricle function (i.e. right ventricle fractional area change, tricuspid annular plane systolic excursion and tricuspid systolic velocity) in the quantification of even reversible or subclinical right ventricular regional non-uniformity caused by acute right ventricle pressure overload [8]. However, accurate echocardiographic imaging of the right ventricle free wall can be technically challenging and other imaging modalities, such as cardiac computed tomography (CT), might be a worthwhile option for assessment of right ventricle dysfunction. Although there is no consensus on which is the best marker of right ventricle dysfunction, HeNZler et al. [9] proposed right ventricle/left ventricle volume as the most accurate CT parameter, and its combination with N-terminal pro-BNP or troponin I measurements seemed to further improve the diagnostic accuracy of either test alone. Notwithstanding, proper evidence demonstrating that CT parameters can add independent prognostic value to traditional PE risk schemes is lacking.

Despite the existence of several risk scores, accurate prediction of PE prognosis remains a challenge. The risk scores are not fully accomplishing their primary goal of supporting the attending physician in important clinical decisions (suitability for outpatient management or thrombolysis for high-risk normotensive cases). The number of PE risk schemes continues to proliferate, often comprising a large number of variables (e.g. PESI has 11 variables and LR-PED includes eight) some of which are not always available or easily collectable, which somewhat limits their application in busy daily practice. Possibly, research should aim to develop comprehensive cardiovascular risk assessment tools that could aid clinicians without overwhelming them with the sheer volume of data available on this subject. With this in mind, we tested a renowned acute 
coronary syndrome risk model, the Global Registry of Acute Coronary Events (GRACE), in a PE cohort, as it contains important haemodynamic variables and target organ lesion markers providing an interesting assessment of illness severity. Although the results warrant further validation in independent cohorts, the GRACE seemed to accurately capture PE severity [10].

Acute PE is a complex condition with several organ-interactions, a broad clinical presentation and a wide prognosis. Currently, PE risk stratification schemes may perform satisfactorily at ruling-out illness severity and allow assessment of patient eligibility for outpatient management. The next step would be to correctly recognise truly high-risk normotensive $\mathrm{PE}$ cases that would benefit from early and more aggressive therapies, such as thrombolysis.

@ERSpublications

The next step for PE risk stratification will be to correctly recognise truly high-risk normotensive PE cases http://ow.ly/nSvxM

Luis Paiva, Sérgio Barra and Rui Providência

Coimbra's Hospital and University Centre, Coimbra, Portugal.

Correspondence: L. Paiva, Quinta dos Vales, 3041-801, S. Martinho do Bispo, Portugal. E-mail: luisvpaiva@gmail.com

Received: Feb 262013 | Accepted after revision: March 052013

Conflict of interest: None declared.

\section{References}

Sanchez O, Trinquart L, Planquette B, et al. Echocardiography and pulmonary embolism severity index have independent prognostic roles in pulmonary embolism. Eur Respir J 2013; 42: 681-688.

2 Aujesky D, Obrosky DS, Stone RA, et al. Derivation and validation of a prognostic model for pulmonary embolism. Am J Respir Crit Care Med 2005; 172: 1041-1046.

3 Sam A, Sánchez D, Gómez V, et al. The shock index and the simplified PESI for identification of low-risk patients with acute pulmonary embolism. Eur Respir J 2011; 37: 762-766.

4 Moores L, Zamarro C, Gómez V, et al. Changes in PESI scores predict mortality in intermediate-risk patients with acute pulmonary embolism. Eur Respir J 2013; 41: 354-359.

de Lemos JA, McGuire DK, Drazner MH. B-type natriuretic peptide in cardiovascular disease. Lancet 2003; 362: 316-322. Barra S, Paiva L, Providência R, et al. LR-PED rule: low risk pulmonary embolism decision rule - a new decision score for low risk pulmonary embolism. Thromb Res 2012; 130: 327-333.

7 Barra SN, Paiva LV, Providência R, et al. Atrial fibrillation in acute pulmonary embolism: prognostic considerations. Emerg Med J 2013 [In press DOI: 10.1136/emermed-2012-202089].

8 Takamura T, Dohi K, Onishi K, et al. Reversible left ventricular regional non-uniformity quantified by speckletracking displacement and strain imaging in patients with acute pulmonary embolism. J Am Soc Echocardiogr 2011; 24: 792-802.

9 Henzler T, Roeger S, Meyer M, et al. Pulmonary embolism: CT signs and cardiac biomarkers for predicting right ventricular dysfunction. Eur Respir J 2012; 39: 919-926.

10 Paiva LV, Providência RC, Barra SN, et al. Cardiovascular risk assessment of pulmonary embolism with GRACE risk scorê. Am J Cardiol 2013; 111: 425-431.

\section{Smoking resumption after lung transplantation: standardised screening and importance for long-term outcome}

To the Editor:

Worldwide, about $40 \%$ of lung transplantations (LTx) are performed for end-stage emphysema [1]. Eligible patients are enrolled on the waiting list after at least 6 months of smoking cessation [1]. Although in most centres smoking behaviour after LTx is not routinely monitored, resuming smoking can complicate posttransplant outcome [2-5].

In general, smoking relapse can be found in $12-40 \%$ of all liver, heart and renal transplant patients [3]. Smoking is mostly assessed by use of a questionnaire. Only the study of BoTHA et al. [4] combined a questionnaire with urinary cotinine detection. We previously reported post-LTx smoking in $11 \%$ of our 